\title{
Efecto del consumo de extracto de alfalfa (Medicago sativa $L$.) sobre el recuento de leucocitos, en ratones (Mus musculus)
}

\author{
Effect of the consumption of alfalfa extract (Medicago sativa $L$.) on the leukocyte count, in mice (Mus \\ musculus)
}

Jony Amaro 1,a, María Elena Iparraguirre 1,b

\section{RESUMEN}

La alfalfa, Medicago sativa $L$, es una leguminosa altamente ecológica, de fácil cultivo y comercialización. En Perú, se cultiva como forraje para animales. Objetivos: Determinar el efecto del consumo del extracto de alfalfa sobre el recuento de leucocitos, en ratón albino. Material y métodos: Estudio experimental, realizado en el Bioterio de la Facultad de Farmacia y Bioquímica de la Universidad Nacional Mayor de San Marcos. Se utilizaron veinte ratones albinos de la cepa balb/c, machos, de peso promedio $23,2 \pm 32,8 \mathrm{~g}$. Se formaron dos grupos: experimental y control, cada uno, de diez ratones. Al grupo experimental se le administró, vía oral por canulación extracto de alfalfa, en dosis de $250 \mathrm{mg} / \mathrm{kg}$, volumen de $2 \mathrm{ml}$, y al grupo control, también, vía oral por canulación, placebo, volumen de $2 \mathrm{ml}$, cada cinco horas, durante una semana, alternando con nutrientes estándar. Se realizó recuento de leucocitos. Resultados: Dentro del periodo de acondicionamiento, se observó un ligero incremento en la media de leucocitos del grupo control $(3940 \pm 1549,3)$ frente al grupo experimental $(3840 \pm 1468,3)$, cuya diferencia no fue significativa. Luego de administrar al grupo experimental el extracto de Medicago sativa L., alternando con sus nutrientes respectivos, se observó diferencia significativa de la media $(7800 \pm 2885,2)$ frente al grupo control $(3930$ $\pm 1518,1)(\mathrm{p}<0,05)$. Conclusiones: En condiciones experimentales, el consumo del extracto de Medicago sativa $L$. incrementó significativamente el número de leucocitos.

PALABRAS CLAVE: Medicago sativa, citocinas, inmunomodulación, flavonoides, inmunidad humoral, inmunidad celular. (Fuente: DeCS BIREME).

\section{SUMMARY}

Alfalfa, Medicago sativa L, is a highly organic legume, easy to grow and commercialize. In Peru, it is cultivated as fodder for animals. Objectives: To determine the effect of alfalfa extract consumption on the white blood cell count in albino mouse. Methods: Experimental study. carried out in Bioterio of the Facultad de Farmacia y Bioquímica of Universidad Nacional Mayor de San Marcos. Twenty albino mice of the balb / c strain, male, of average weight 23.2 $\pm 32.8 \mathrm{~g}$ were used. Two groups were formed: experimental and control, each of ten mice. The experimental group was administered, orally by cannulation alfalfa extract, in a dose of $250 \mathrm{mg} / \mathrm{kg}$, volume of $2 \mathrm{ml}$, and the control group, also, orally by cannulation, placebo, volume of $2 \mathrm{ml}$, every five hours, for a week, alternating with standard nutrients. Leukocyte count was performed. Results: Within the conditioning period, there was a slight increase in leukocytes mean of the control group ( $3940 \pm 1549.3)$ compared to the experimental group ( $3840 \pm 1468.3)$, whose

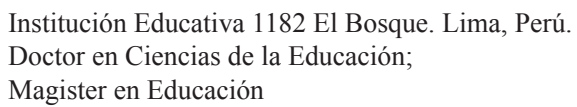


difference was not significant. After administering the extract of Medicago sativa $L$ to the experimental group, alternating with their respective nutrients, a significant difference was observed between the mean $(7800 \pm 2885.2)$ and the control group $(3930 \pm 1518.1)(\mathrm{p}<0.05)$. Conclusions: Under experimental conditions, the consumption of Medicago sativa extract significantly increased the number of leukocytes.

KEYWORDS: Medicago sativa, cytokines, immunomodulation, flavonoids, humoral, immunity, cellular. (Source: MeSH NLM).

\section{INTRODUCCIÓN}

La alfalfa, Medicago sativa $L$, es una leguminosa altamente ecológica, de fácil cultivo y comercialización. En el país, se cultiva como forraje para animales; sin embargo, nuestra población de escasos recursos utiliza de manera esporádica y empírica el jugo extraído de las hojas frescas de alfalfa como una alternativa para disminuir la anemia, combatir la tuberculosis, en niños, gestantes y adultos. En otros países, se ha elaborado alimentos que tienen como ingredientes un extracto de hojas de alfalfa, rico en macronutrientes (proteínas, azúcares y lípidos) y micronutrientes (vitaminas y oligoelementos) (1).

Asimismo, estas plantas producen un gran número de moléculas, entre ellas, los metabolitos secundarios que son sintetizados para diferentes propósitos, incluyendo regulación del crecimiento, interacciones intra e interespecíficas y defensa contra depredadores e infecciones; estas sustancias tienen un potencial inmunoestimulante y antimicrobiano en los seres humanos. Dentro de estos metabolitos se incluyen los flavonoides, indoles, fitoesteroles, polisacáridos, sesquiterpenos, alcaloides, glucanos, taninos, entre otros (2).

Los metabolitos secundarios producidos por las plantas son utilizados por el hombre para tratar una variedad de enfermedades. Aproximadamente el 25\% de las drogas prescritas en el mundo provienen de plantas y se ha estimado que $60 \%$ de las drogas antiinfecciosas y anti-tumorales son de origen natural (3). Muchos de estos metabolitos secundarios presentan actividades farmacológicas y biológicas interesantes y son usados como agentes quimioterapéuticos o sirven como punto inicial para el desarrollo de medicinas (4).

Por otro lado, el sistema inmune es el encargado de defender al organismo de los agentes agresores que se encuentran a nuestro alrededor. El sistema inmune debe mantener su equilibrio y capacidad de respuesta, ya que su alteración puede ser la base de un importante número de enfermedades (5). La modulación de la respuesta, ya sea estimulándola o suprimiéndola, puede ayudar a mantener un estado libre de enfermedades. La búsqueda de agentes inmunomoduladores que equilibren el sistema inmune ha suscitado interés durante mucho tiempo (6).

Muchas de las plantas utilizadas en la medicina tradicional han demostrado poseer actividades inmunomoduladoras, mejorando considerablemente los mecanismos inmunológicos encargados de combatir las células malignas y proteger al organismo de las infecciones $(7,8)$.

El objetivo de este estudio fue determinar la influencia del extracto de Medicago sativa sobre el recuento de leucocitos, en ratones albinos.

\section{MATERIAL Y MÉTODOS}

Estudio de tipo experimental, ejecutado en el Bioterio de la Facultad de Farmacia y Bioquímica de la Universidad Nacional Mayor de San Marcos. Se utilizaron 20 ratones albinos de la cepa Balb/c, machos, de 23,2 $\pm 32,8$ g., de peso promedio, procedentes del bioterio del Instituto Nacional de Salud (Lima, Perú).

Fueron mantenidos en jaulas individuales a temperatura ambiente de $22 \pm 2{ }^{\circ} \mathrm{C}$, con ciclo luz/ oscuridad de 12 horas, con alimentos y agua adlíbitum. Luego de la aclimatación de 5 días, fueron distribuidos aleatoriamente en dos grupos de 10 ratones cada uno: grupo experimental y grupo control. Al grupo experimental, se administró por vía oral y canulación, el extracto de Medicago sativa, volumen de $2 \mathrm{ml}$, en dosis de $250 \mathrm{mg} / \mathrm{kg}$, cada cinco horas, durante una semana, alternando con nutrientes estrictamente balanceados procedentes del Instituto Nacional de Salud, en dosis y tiempos de acuerdo a normas estándar. Al grupo control, se administró, por vía oral y canulación, de placebo (solución suero fisiológico), volumen de $2 \mathrm{ml}$, cada cinco horas durante una semana, alternando con nutrientes estrictamente balanceados. 
Después del proceso de aclimatación de 5 días se hizo la pre-medición. Se extrajo las muestras de sangre de la vena safena de la cola, de acuerdo al método de Ciriaco et al (9), en volumen de 0,15 mililitros para evaluar el número de leucocitos de ambos grupos. Se utilizó el método de fórmula leucocitaria Schilling y la técnica frotis con coloración de fijación Wright. Además, se utilizó como anticoagulante EDTA, de acuerdo al procedimiento del Laboratorio de Análisis Clínico de la Facultad de Farmacia y Bioquímica de la Universidad Nacional Mayor de San Marcos; luego de aplicar el experimento, se realizó nueva medición.

Se utilizó la planta entera de Medicago sativa $L$, del Distrito de Ambo, Provincia Huánuco, Departamento de Huánuco, adquirido en el mercado 'Santa Rosa', San Juan de Lurigancho. Las hojas y tallos frescos se cortaron en pequeños trozos que fueron secados, molidos y tamizados hasta obtener un polvo muy fino que se empleó para preparar el extracto acuoso.

Se elaboró el extracto acuoso de la alfalfa, de acuerdo al protocolo descrito por Gonzales-Gonzales-Carazas et al (10). Para el tratamiento de los animales con el extracto, se elaboró la solución a una concentración de $250 \mathrm{mg} / \mathrm{kg}$, diluyéndolo en agua bidestilada, y se conservó a $-4^{\circ} \mathrm{C}$; se preparó las dosis en volúmenes de $2 \mathrm{ml}$.

La identificación del metabolito secundario, flavonoides, se realizó utilizando la técnica de ensayo de Shinoda en la muestra vegetal Medicago sativa, elaborada por la Unidad de Investigación en Productos
Naturales de la Universidad Peruana Cayetano Heredia.

Los datos cumplieron las premisas de normalidad (Kolmogorov-Smirnov) y homogeneidad de varianza (Prueba de Levene) $(\mathrm{p}>0,05)$, por lo cual todos los resultados fueron sometidos a análisis de media, seguido de una prueba $t$ de student, para buscar diferencias significativas.

Se determinó la media y la desviación estándar. Se consideró, estadísticamente significativo un $\mathrm{p}<0,05$. Para los análisis se utilizó el programa MS Excel v 2013.

El estudio fue manejado de acuerdo con el protocolo de la Comisión Ética para la Experimentación Animal de la Universitat de les Illes Balears (11).

\section{RESULTADOS}

El estudio fitoquímico preliminar, utilizando la prueba Shinoda, mostró la presencia de flavonoides, en el extracto de Medicago sativa.

Después del periodo de acondicionamiento, en el tratamiento experimental, el recuento de leucocitos, en el grupo control, fue $3940 \pm 1549,3$ mientras en el grupo experimental $3840 \pm 1468,3(\mathrm{p}>0,05)$ (Gráfico 1). Asimismo, después de la administración del extracto de Medicago sativa, el recuento de leucocitos mostró $(7800 \pm 2885,2)$ frente al grupo control $(3930$ $\pm 1518,1),(\mathrm{p}<0,05)($ Gráfico 2).

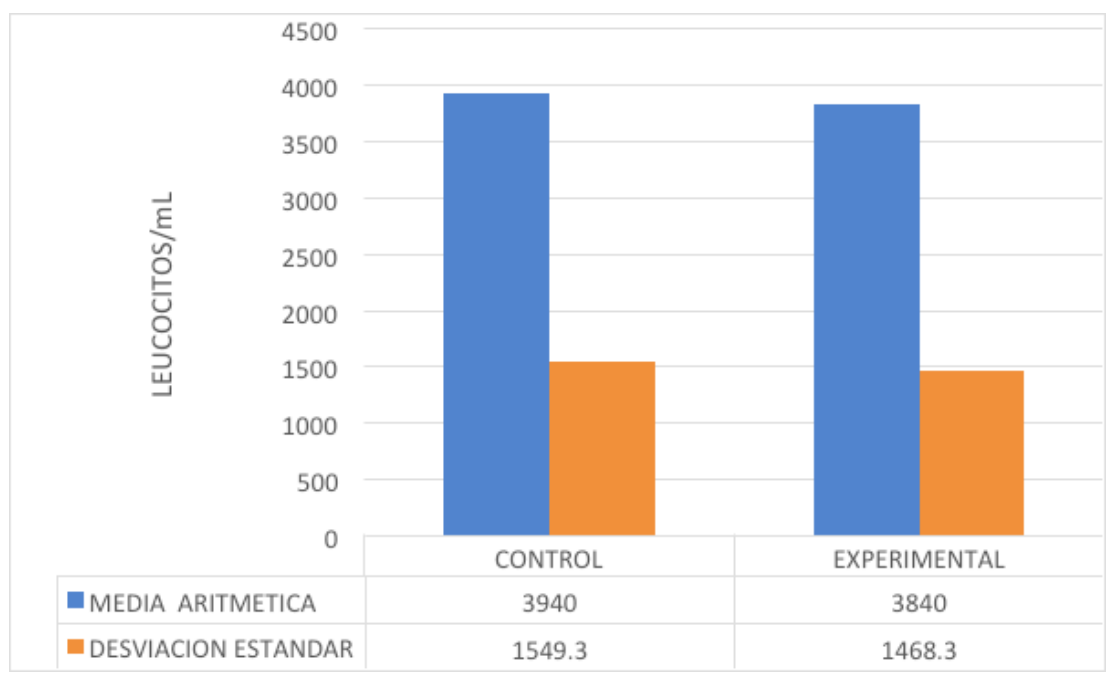

Gráfico 1. Efecto del consumo de nutriente balanceado y agua, durante el periodo de acondicionamiento, en ambos grupos. 


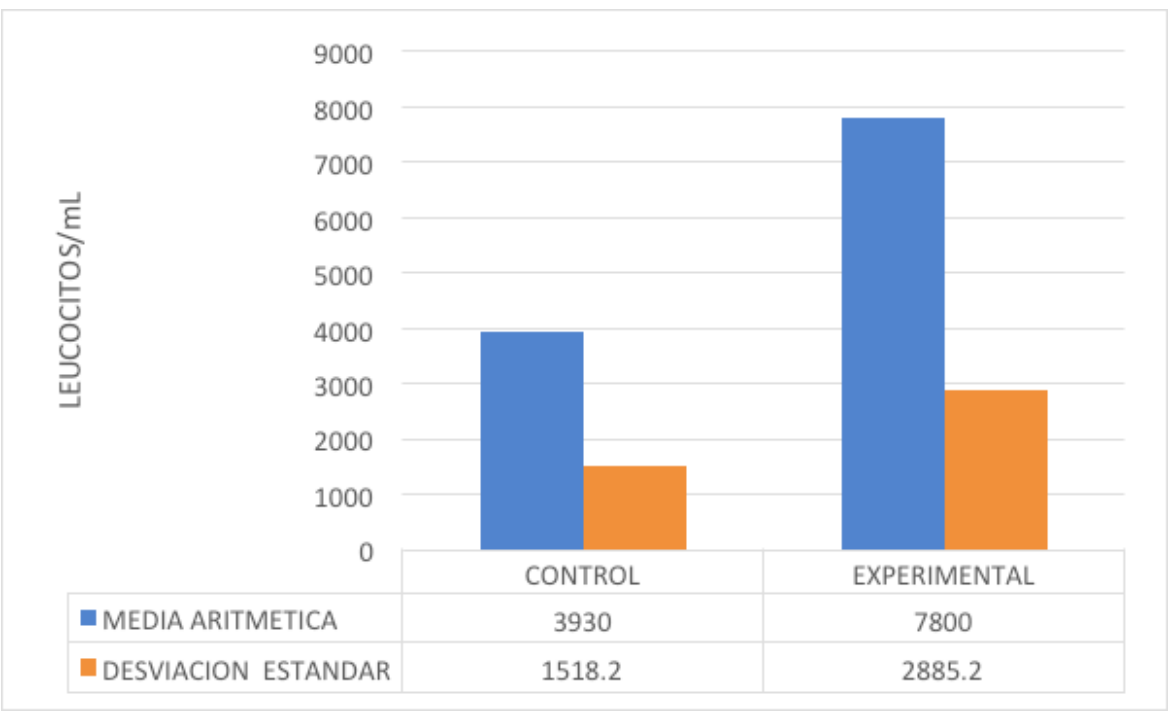

Gráfico 2. Efecto del consumo de extracto de Medicago sativa, alternando con el nutriente, durante una semana (grupo experimental), y placebo (grupo control).

\section{DISCUSIÓN}

Se observó que el consumo de alfalfa, en forma de extracto, produjo incremento de leucocitos.

García J (12), encontró incremento de leucocitos con extracto de maca (Lepidium peruvianum) de tres tipos -extracto clorofórmico (EC), extracto acuoso (EAc) y extracto metanólico (EM)- y lo relacionó con alto contenido en saponinas, alcaloides y flavonoides, el estudio elaboró tres niveles de extracto y el mayor nivel de leucocitos observó con extracto clorofórmico de maca.

El estudio realizado por Dina Torres identificó el incremento del sistema inmunológico en el extracto de maca (Lepidium peruvianum) y lo relacionó con su alto contenido en saponinas, alcaloides y flavonoides (13). El estudio elaboró, también, tres niveles de extracto: extracto clorofórmico (EC), extracto acuoso (EAc) y extracto metanólico (EM), y el mayor nivel de leucocitos fue con el extracto metanólico de maca.

Asimismo, Álvarez E (14), identificó el incremento de leucocitos en el extracto de maca (Lepidium peruvianum) y lo relacionó con su alto contenido en flavonoides. En el estudio trabajó solo con extracto metanólico de maca (EM) de tres variedades: blanco, morado y rojo, y en las tres variedades se observó el incremento de leucocitos.

El estudio realizado por Amaro, identificó el incremento de leucocitos en el extracto de betarraga y habas, y los relacionó con su alto contenido en flavonoides $(15,16)$.

Además, Jung et al, observaron en los animales expuestos / no expuestos a la radiación se sacrificaron en los días de radiación 10 y 30, la proliferación de esplenocitos (leucocitos), que se redujo después de la irradiación, se aumentó significativamente mediante la suplementación con quercetina (flavonoide) después de 30 días de irradiación (17).

Kamboh et al (18), observaron que la suplementación de la dieta basal de pollos de engorde, ya sea con genisteína o hesperidina (flavonoides), mejora el estado inmunológico. Asimismo, Pinar et al (19), observaron también en compuestos $1,2,3$ y la fracción total de flavonoides, un efecto significativo inmuno-estimulante sobre los neutrófilos (19).

Por otro lado, el análisis fitoquímico de la alfalfa, identificó la presencia de flavonoides, metabolito secundario que explicaría, probablemente, el incremento de leucocitos, de acuerdo a lo reportado por otros autores, aunque no se descarta la presencia de otros metabolitos.

En conclusión, el consumo de extracto de Medicago sativa incrementa, significativamente, el recuento de leucocitos, en un modelo experimental; estos resultados proveen las primeras evidencias para el consumo de la alfalfa como alimento inmunoestimulador. 


\section{Declaración de financiamiento y de conflictos de intereses:}

El estudio fue financiado por los autores. Declaran no existe conflictos de interés

\section{Contribución de autoría:}

JA: Diseño del estudio, análisis de los datos, redacción, revisión crítica importante del contenido del manuscrito, aprobación de la versión que se publicará; MA: Recolección de datos

\section{Correspondencia:}

Jony Amaro

Mz B1 lote 14 Sargento Lorenz San Juan Lurigancho. Lima, Perú.

Teléfono: 5114555186 / 51955119117

Correo electrónico: Jony200927@live.com

\section{REFERENCIAS BIBLIOGRAFICAS}

1. Shah FH, Salam Sheikh A, Farrukh N, Rasool A. A comparison of leaf protein concentrates fortified dishes and milk as supplements for children with nutritionally inadequate diets. Plan Foods in Human Nutrition. 2003; 30:245-58.

2. WilliamsJ.Review ofantiviral and immunomodulating properties of plants of the Peruvian rainforest with a particular emphasis on uña de gato and sangre de grado. Altern Med Rev. 2001; 6(6):567-79.

3. Kirszberg C, Esquenazi D, Celuta A, Rumjanek V. The effect of a catechin-rich extract of cocosnucifera on lymphocytes proliferation. Phytother Res. 2003; 17:1054-8.

4. Verpoorte R. Exploration of nature's chemodiversity: the role secondary metabolites as leads indrug development. Drug Develop Trends. 1998; 3:232-8.

5. Abbas A, Lichtman A, Pober J. Inmunología celular molecular. 3era ed. Madrid: McGraw HillInteramericana; 2002.

6. Dahanukar SA, Kulkarni RA, Rene NN. Pharmacology of medicinal plants and natural products. Indian J Pharmacol. 2000; 32:81-118.

7. Atal CK, Sharma ML, Kaul A, Khajuria A. Immunomodulating agents of plant origin I: Preliminary screening. J Ethnopharmacol. 1989; 18(2):133-41.

8. Perez JE, Isaza G, Bueno JG, et al. Efecto de los extractos de Phenaxrugosus, Tabebuian Chrysantha, Althernantera Williamsii y Solanum Dolichosepalum sobre el leucograma y la producción de anticuerpos en ratas. Rev Med Risaralda. 2004; 10(2):13-21.
9. Ciriaco A, Mendiola J, Falcon D, Cantillo J. Obtención de sangre e inyección intravenoso en roedores por vía de la vena safena. Animales de Laboratorio. 1999; 33:6-7. (Citado el 8 de marzo del 2017) Disponible en: http://www.oc.lm.ehu.es/Fundamentos/ doctorado/cursos/CirExp/PincharSafena.pdf

10. Gonzales-Carazas EF, Melgarejo-García GC, Chávez-Conde LK, et al. Efecto terapéutico del extracto etanólico de Erythroxylum coca spp. en anemia ferropénica inducida en ratas Holtzman macho. An Fac med. 2013;74(1):7-10.

11. Uiw. Comité de Ética de Experimentación Animal (CEEA/AAEB).[portal en internet]. Islas Baleares: Universitat de les Illes Balears; 5 de noviembre del 2013. [citado el 3 de abril 2017]. Disponible en: http:// www.uib.es/es/recerca/estructures/comissions/ceea/

12. García J. Comparación de la actividad de extractos de Lepidium peruvianum Chacón (maca) sobre leucocitos procedentes de individuos saludables e infectados con el virus de la inmunodeficiencia humana (HIV). Tesis Profesional. Lima, Perú: Universidad Nacional Mayor de San Marcos; 2010. 180 p.

13. Torres D. Efecto modulador de la respuesta inmune humoral de extractos de Lepidium peruvianum, chacón (maca) en ratones inmunosuprimidos con ciclofosfamida. Tesis Profesional. Lima, Perú: Universidad Nacional Mayor de San Marcos; 2008. $200 \mathrm{p}$.

14. Álvarez EK. Estudio comparativo de la actividad moduladora del extracto metanólico de cuatro ecotipos de Lepidium peruvianum Chacón (maca) sobre la respuesta inmune humoral y celular en ratones. Tesis Profesional. Lima, Perú: Universidad Nacional Mayor de San Marcos; 2008. 250 p.

15. Amaro J. Influencia de la betarraga (Beta vulgaris var. cruenta) en el aumento de leucocitos, en ratones. An Fac med. 2014;75(1):9-12.

16. Amaro J. Efecto del consumo del extracto de haba (Vicia faba L.) en el aumento de leucocitos, en ratones. An Fac med. 2015; 76(4):465-6.

17. Jung JH, Kang JI, Kim HS. Effect of quercetin on impaired immune function in mice exposed to irradiation. Nutr Res Pract. 2012; 6(4):301-7.

18. Kamboh AA, Hang SQ, Khan MA, Zhu WY. In vivo immunomodulatory effects of plant flavonoids in lipopolysaccharide-challenged broilers. Animal. 2016; 10(10):1619-25.

19. Akbay P, Basaran AA, Undeger U, Basaran N. In vitro immunomodulatory activity of flavonoid glycosides from Urtica dioica L. Phytother Res. 2003; 17(1):34-7.

Recibido: 19/03/2017

Aceptado: 14/01/2018 